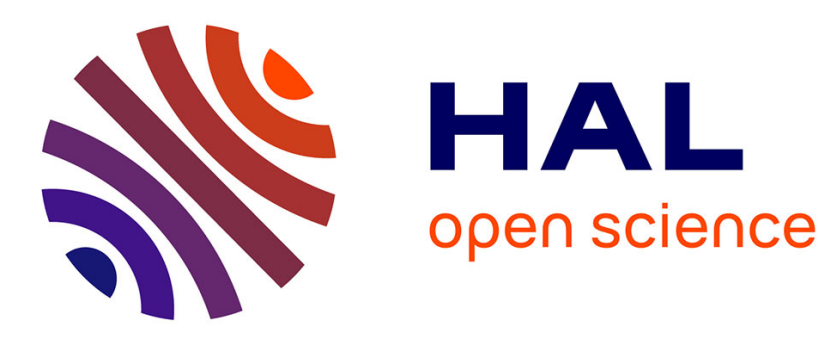

\title{
Cholesteric liquid crystal gels with a graded mechanical stress
}

\author{
Gonzague Agez, Sabrina Relaix, Michel Mitov
}

\section{To cite this version:}

Gonzague Agez, Sabrina Relaix, Michel Mitov. Cholesteric liquid crystal gels with a graded mechanical stress. Physical Review E , 2014, 89 (2), pp.022513. 10.1103/PhysRevE.89.022513 . hal-01730532

\section{HAL Id: hal-01730532 \\ https://hal.science/hal-01730532}

Submitted on 13 Mar 2018

HAL is a multi-disciplinary open access archive for the deposit and dissemination of scientific research documents, whether they are published or not. The documents may come from teaching and research institutions in France or abroad, or from public or private research centers.
L'archive ouverte pluridisciplinaire HAL, est destinée au dépôt et à la diffusion de documents scientifiques de niveau recherche, publiés ou non, émanant des établissements d'enseignement et de recherche français ou étrangers, des laboratoires publics ou privés. 


\title{
Cholesteric liquid crystal gels with a graded mechanical stress
}

\author{
Gonzague Agez, Sabrina Relaix, ${ }^{*}$ and Michel Mitov ${ }^{\dagger}$ \\ Centre d'Elaboration de Matériaux et d'Etudes Structurales, CEMES, CNRS, University Paul-Sabatier, 31055 Toulouse cedex 4, France
}

(Received 8 November 2013; revised manuscript received 29 January 2014; published 28 February 2014)

\begin{abstract}
In cholesteric liquid-crystalline gels, the mechanical role of the polymer network over the structure of the whole gel has been ignored. We show that it is the stress gradient exerted by the network over the helical structure that drives the broadening of the optical band gap, as evidenced by the absence of a gradient in chiral species. Model calculations and finite-difference time-domain simulations show that the network acts as a spring with a stiffness gradient. The present results indicate a revision to the common understanding of the physical properties of liquid-crystalline gels is necessary when a concentration gradient in a polymer network is present.
\end{abstract}

DOI: 10.1103/PhysRevE.89.022513

PACS number(s): 42.70.Df, 61.30.Pq, 81.05.Xj

\section{INTRODUCTION}

\section{A. Motivation of the research}

Liquid crystal (LC) gels, also called polymer-stabilized LCs, are fabricated via UV light polymerization of bifunctional reactive mesogens dissolved with a photoinitiator in a conventional low molar mass LC [1-3]. During the photoreaction, the structure of the LC is copied by the polymer network [4], resulting in a similar morphology which in turn influences the LC structure. Such composite materials have received great interest as a way to stabilize a wide variety of structures for photonic materials with increased functionality, including cholesteric LCs (CLCs) with broad reflection bands [5-15], chiral smectic LCs with controlled nanostructures [16], blue phase chiral LCs with widened temperature ranges $[17,18]$, and CLC films which reflect over $50 \%$ of the light from an unpolarized incident beam [19] or exhibit an electrically controllable omnidirectional propagation of a laser emission [20]. In a nematic LC, the average direction of rodlike molecules is uniform whereas in a CLC the average direction twists along an axis in a periodic helicoidal fashion. As a consequence, a CLC slab may reflect light [21]. At normal incidence (for light propagating along the helical axis), the maximum selective reflection occurs at wavelength $\lambda_{0}$, which is directly related to the helical pitch $p$ by $\lambda_{0}=n p$ where $n$ is the average refractive index $\left[n=\left(n_{o}+n_{e}\right) / 2\right.$, where $n_{o}$ and $n_{e}$ are the ordinary and extraordinary indices or refraction, respectively, and which are measured in directions perpendicular and parallel to the local (uniaxial) director, respectively]. The bandwidth $\Delta \lambda$ is related to the birefringence $\Delta n\left(\Delta n=n_{e}-n_{o}\right)$ and $p$ by: $\Delta \lambda=p \Delta n$. The reflection is selective, meaning $\Delta \lambda$ is limited to a few tens of nanometers in the visible spectrum as the birefringence is typically limited to 0.5 . However, CLCs with helical pitch gradients have to be utilized in novel applications [22] for which an enlarged color gamut for light reflection is required. Intense research activities have thus focused on finding methods to design broad band reflective CLCs (see [23] for a review). A large effort was directed towards the generation of a concentration gradient of chiral species coupled to a gradient in a polymer network from which a pitch gradient is expected [5-15]. A UV

\footnotetext{
*Present address: Apple, Cuppertino, CA

${ }^{\dagger}$ Corresponding author: mitov@cemes.fr
}

gradient was generated during the polymerization due to the incorporation of a UV absorber [5] or the natural UV absorbing properties of CLCs [9]. As a consequence, the gel existed in a cholesteric network with a concentration gradient embedded in a nonreactive CLC. All discussions on the generation of a broadened reflection band have focused on the generation of a gradient in chiral species that follows the concentration gradient in the network.

\section{B. Scope and aims of the present study}

This paper addresses the mechanical role of the polymer network on the cholesteric structure of the whole gel, the role of which has been ignored in the literature. We show that the graded mechanical stress, induced by the network, on the helical structure of the CLC is fundamentally responsible for the generation of a pitch gradient and subsequent broadening of the band gap. The physics of light propagation in a CLC gel with a polymer network that is asymmetrically distributed has to be fully understood to optimize the system's properties. The present work contributes to this challenging topic by considering the basic mechanical role of the structure-gradient polymer network over the CLC, enabling a new understanding of the optical behavior of the gel. The present results suggest that a revision to the understanding of the physical properties of CLC gels is necessary when a concentration gradient is present in the polymer network.

For our demonstration, we used two different premixtures. Premixture I was made with reactive and nonreactive mesogens which were both chiral. We demonstrate the broadening of the band gap in the absence of any gradient in chiral species. Then, premixture II was made with achiral mesogenic monomers and a chiral CLC; this mixture corresponds to the situation commonly found in the literature. The structural study of the polymer network by transmission electron microscopy (TEM) provided the basis for modeling the gel structure like a coil spring with a gradient of spring constants acting on the helical structure of the CLC in which the coil spring is embedded. The model generates a pitch gradient from a stress gradient, which arises from the stiffness gradient of the coil spring as evidenced from the spatial distribution of the network when directly observed by TEM. This model represents a conceptual advance in the field of LC gels. A finite-difference time-domain (FDTD) simulation software [24] was used to solve Maxwell's equations within the theoretical structure. 


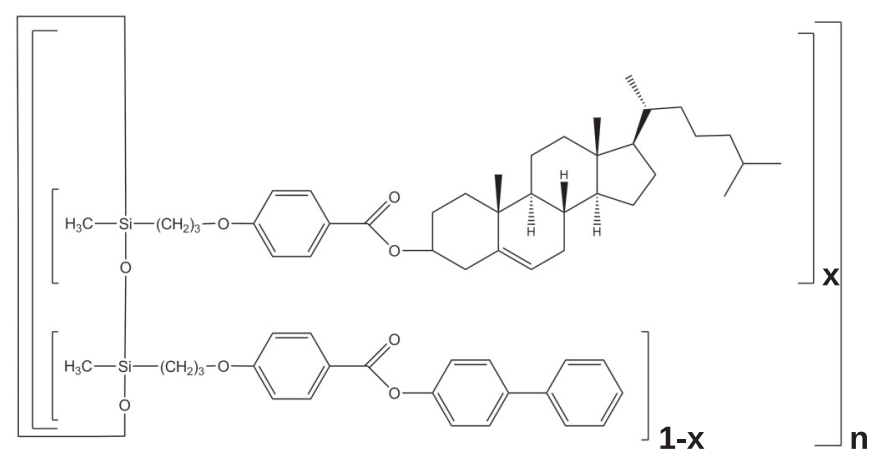

(a)

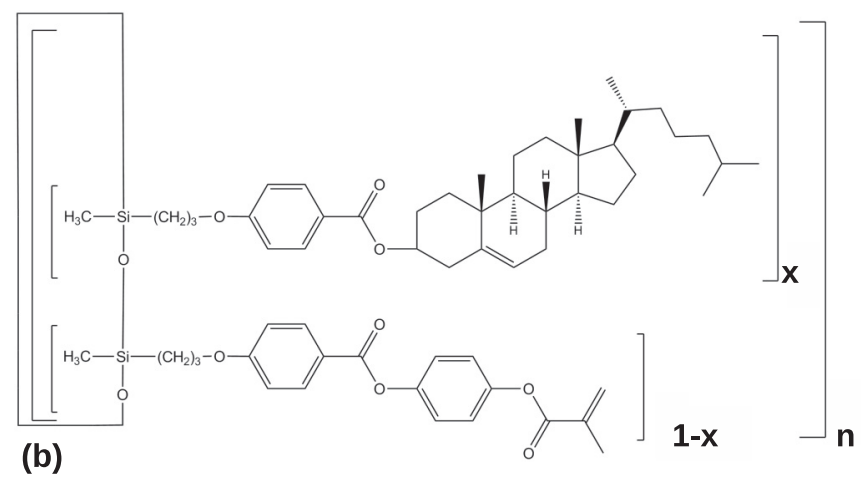

FIG. 1. Chemical structures of cholesteric oligomers used in premixture I (images courtesy of E. Hanelt). (a) Nonreactive oligomer. (b) Photopolymerizable and crosslinkable oligomer. $x$ is the molar fraction of the chiral mesogen and $n$ represents the ring size of the siloxane backbone. $x=0.31$ and $n=4$ for both compounds.

Very good agreement between the simulated transmission or reflection spectra and the experimental data gives credit to this model where the gel structure acts as a spring with a graded stiffness in which the nonreactive LC is embedded.

\section{METHODS}

Premixtures. Premixture I was composed of $10 \mathrm{wt} \%$ photocrosslinkable (RMR) and 90 wt \% nonphotocrosslinkable (SR) cyclosiloxane oligomers (from Wacker) [25] which exhibit a cholesteric phase (in the range $55-170^{\circ} \mathrm{C}$ ) with very similar chemical structures (Fig. 1) [26]. RMR and SR are single molecules, not mixtures. Irgacure 907 (from Ciba-Geigy) was added ( $2 \mathrm{wt} \%$ compared to the RMR amount) as the photo-initiator. When inhibition of thermally induced polymerization was desired, $500 \mathrm{ppm}$ of phenotiazine (PTZ, from Fluka) was added. Premixture II was composed of a CLC (BL094, from Merck Ltd.) mixed with bifunctional photoreactive nematic mesogens (RM257, from Merck Ltd.) at a concentration ratio of $91 \mathrm{wt} \% \mathrm{LC}$ to $9 \mathrm{wt} \%$ monomer, along with photoinitiator Irgacure 907 (2 wt \% compared to the RM257 amount).

Cells. Premixture I: Cells were made from two glass substrates separated by $20-\mu \mathrm{m}$ spacers. No surface alignment layer was used. Premixture II: Cells were made from two pieces of indium-tin-oxide coated glass substrates bonded together with glue containing $6-\mu \mathrm{m}$ spacers. The surfaces were treated with polyimide, which was rubbed to induce planar alignment.
UV exposure. Premixture I: UV (centered at $365 \mathrm{~nm}$ ) exposure was carried out at $130{ }^{\circ} \mathrm{C}$ for 50 min with an intensity of $0.05 \mathrm{~mW} / \mathrm{cm}^{2}$. Premixture II: UV (centered at $365 \mathrm{~nm}$ ) exposure was carried out at room temperature (RT) for $30 \mathrm{~min}$ with an intensity of $0.10 \mathrm{~mW} / \mathrm{cm}^{2}$. For both premixtures, a black substrate was deposited below the cell when asymmetrical conditions were promoted.

Optics. The transmittance and reflectance spectra of films were collected at RT, at normal incidence, and with unpolarized incident light. Depending on the wavelength range desired for the investigation, an HR2000CG-UV-NIR spectrophotometer (from Ocean Optics) associated with an optical microscope $(\lambda=400-900 \mathrm{~nm})$ or a Perkin-Elmer IR spectrometer Spectrum $100(\lambda=900-2500 \mathrm{~nm}$ [Fig. S5]) was used. Baselines were recorded with air (respectively, a mirror) in the beam path for transmittance (respectively, reflectance) measurements.

Sample preparation for SEM. The film of premixture I was solid at RT (it is a cross-linked polymer network embedded in a quenched oligomer film in the glassy state). The cell was broken in a direction perpendicular to the substrates. This cross section of the material was then coated with a 2-nm-thick gold film to create a conductive surface and it was finally fixed on a support with a carbon-coated adhesive.

Sample preparation for TEM. Resin was substituted for the LC component by immersing the gel cell in a bath of LR White Resin (from the London Resin Company) diluted in a solvent (cyclohexane and then methanol). The concentration of resin in the solvent was progressively increased from one bath to the next to gently optimize the filling of the network pores. Although some fragile parts of the network (free oligomers) are expected to be lost in the course of this process, this method allows us to present the network's spatial distribution. The resin polymerization occurred at $80^{\circ} \mathrm{C}$. After polymerization, the cell was opened at RT. The polymer network embedded in the resin matrix was removed from the substrates and embedded in a second (epoxy) resin. This composite was then cut into 80-nm-thick slices in a direction perpendicular to the surface with an ultramicrotome (UltraCut S from Reichert) at RT. The slices of cross sections were retrieved on carbon-coated grids for TEM observations.

Transmission electron microscopy. The cross-sectional cuts of cholesteric polymer network embedded in resin were observed with a Philips CM12 TEM.

Scanning electronic microscopy. JSM-6490 scanning electron microscope (SEM) from JEOL was used (secondary electron imaging mode at $20 \mathrm{kV}$ under normal conditions).

Analysis of TEM images. TEM images of cross sections were used to extract the network concentration profiles. A threshold selection method from a gray-level histogram was used to differentiate the network from the resin matrix. Details are given in Fig. S1 of the Supplemental Material [27]. Network density profiles were calculated from cross-sectional images that were $6 \times 120 \mu \mathrm{m}^{2}$ in size. The resolution was 120 pixels per $\mu \mathrm{m}$.

Calculations. The spring model (cf. Fig. 5) utilizes a series of $N$ springs placed end to end. We assumed that every subspring has the same relaxation length $l_{0}$, so the length of the whole spring is $L_{0}=N l_{0}$. The network with a concentration gradient is simulated by a series of $N$ springs with gradual stiffness $k_{i}$ (with $i$ from 1 to $N$ ). The stressed spring $i$ with a 
(a)

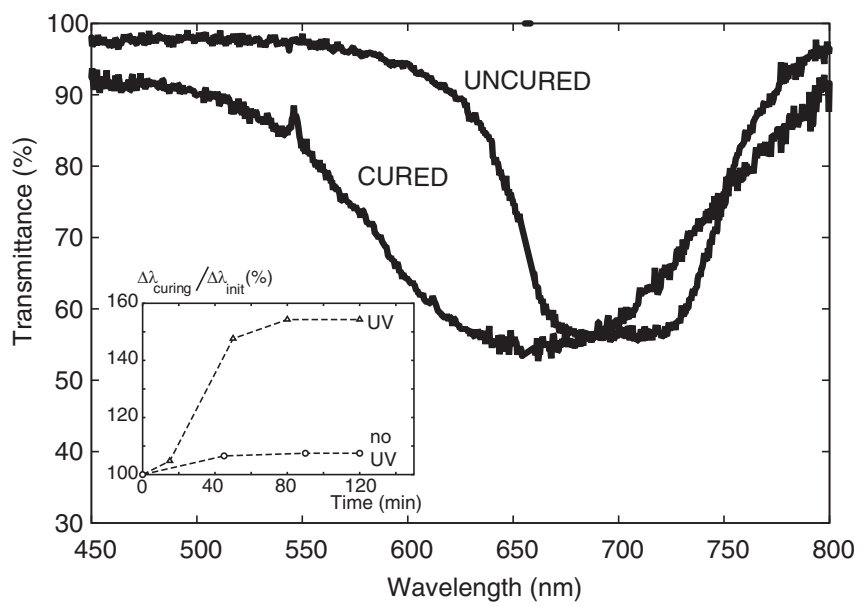

(b)

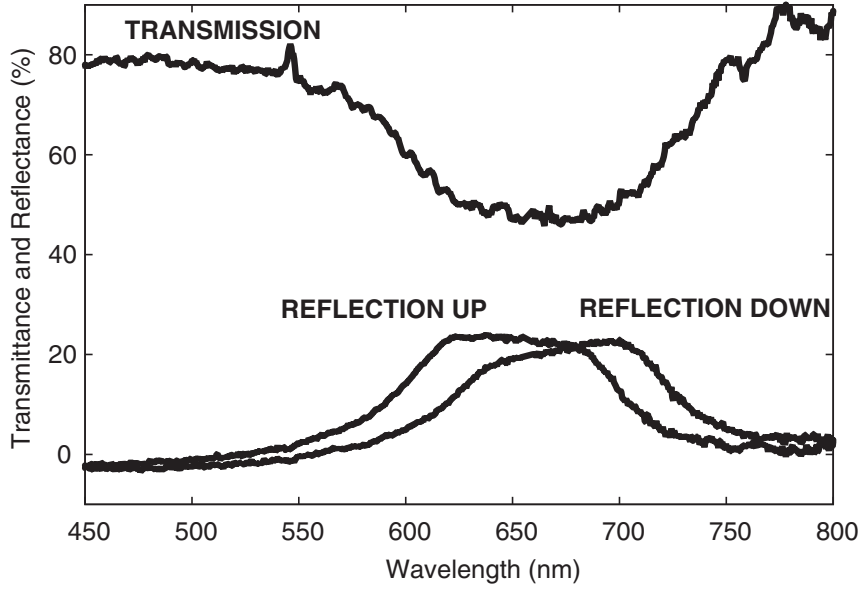

FIG. 2. (a) Transmittance of a $20-\mu \mathrm{m}$-thick film of premixture I both uncured and cured for 50 minutes under asymmetrical conditions. Inset: Variation of the relative bandwidth $\Delta \lambda / \Delta \lambda_{0}$ with time. $\Delta \lambda$ (respectively, $\Delta \lambda_{0}$ ) is the full width at half maximum of the band gap after (respectively, before) polymerization. The time evolution is investigated in the absence of UV and with UV under asymmetrical conditions. A plateau is reached after approximately $80 \mathrm{~min}$. (b) Transmittance, and reflectance from the UP and DOWN sides of the cell, after 120 min of irradiation. The UP side corresponds to the side of the incoming UV beam.

length $l_{i}$ exerts a force $F_{i}^{R}$ on the spring $i-1$ toward the right and a force $F_{i}^{L}$ on the spring $i+1$ toward the left. At the steady state, according to Hooke's law, the force equilibrium can be written for $\mathrm{i}=1$ to $N-1$ with the following equation:

$$
\begin{aligned}
F_{i}^{L}=F_{i+1}^{R} & \Rightarrow k_{i}\left(l_{i}-l_{0}\right)=k_{i+1}\left(l_{i+1}-l_{0}\right) \\
& \Rightarrow x_{i}=\left(\frac{k_{i+1}}{k_{i}}\right) x_{i+1} .
\end{aligned}
$$

The sum of the subspring elongations $x_{i}=l_{i}-l_{0}$ for $N$ springs is equal to the whole elongation:

$$
\sum_{i=1}^{N} x_{i}=L-L_{0}
$$

where $L$ is the thickness of the gel layer. From recurrence relation (1), we deduce

$$
x_{i}=\left(\frac{k_{N}}{k_{i}}\right) x_{N} \text { leading to } \sum_{i=1}^{N} x_{i}=\sum_{i=1}^{N}\left(\frac{k_{N}}{k_{i}}\right) x_{N} .
$$

By identification with relation (2), the final term of the series is obtained:

$$
x_{N}=\frac{L-L_{0}}{\sum_{i=1}^{N}\left(\frac{k_{N}}{k_{i}}\right)} .
$$

If the distribution of spring constants $k_{i}$ is known, all the elongations from $x_{1}$ to $x_{N}$ can be calculated.

Simulation methods. We used the FDTD method from the MEEP software package [24]. This electromagnetic simulation software uses Maxwell's equations and let them run over time within some finite computational region. Equations (3) and (4) provide the elongation of each subspring. The local pitch distortion of the cholesteric structure is directly proportional to the elongation (stretching or compression) of the corresponding subspring. The computational grid resolution was 120 pixels per $\mu \mathrm{m}$ (exactly the same resolution as the TEM images). The two-dimension box size was 800 pixels along the direction of light propagation and 360 pixels along the transverse direction. We imposed absorbing boundary conditions (this approach utilizes a system that surrounds the computational cell with a medium that absorbs light without any reflection at all frequencies and angles of incidence). We set the average refractive index $n$ to 1.57 and the birefringence $\Delta n$ to 0.3 . The entire transmission spectrum can then be obtained in a single run via a Fourier transform of the response from a Gaussian short pulse (two runs are needed for normalization).

\section{EXPERIMENT}

\section{A. Experimentum crucis}

The transmission and reflection spectra of premixture I before and after polymerization are shown in Fig. 2.

Premixture I was composed of $10 \mathrm{wt} \%$ photoreactive chiral mesogens and $90 \mathrm{wt} \%$ nonreactive chiral mesogensphotoinitiator mixture. The premixture pouring technique utilized capillary action between two parallel glass substrates (sandwich cell) to distribute the material, forming a $20-\mu \mathrm{m}-$ thick film. The cell exhibited a characteristic planar Grandjean texture at $130^{\circ} \mathrm{C}$ that gave rise to a vividly colored Bragg-like reflection in the red part of the visible spectrum [Fig. 2(a), uncured]. The cell was illuminated with UV from one side to polymerize the reactive mesogens [Fig. 2(a), cured]. The transmission band gap was broadened from the left side of the gap, from 100 to $160 \mathrm{~nm}$ ( $\Delta \lambda$ is currently defined as the full width of the gap at half height). This broadening is not related to the effect of volume shrinkage of the material which results in a blueshift of the reflection band and is well known in cholesteric networks [28] and gels [29]. Since a thermal polymerization could occur at the same time as the photopolymerization, we checked the evolution of the optical properties in the same conditions without UV and with UV when an inhibitor of thermal polymerization is dissolved in premixture I (see Supplemental Material, Fig. S2 [27]); it was found that no band-gap broadening occurs in the former case 


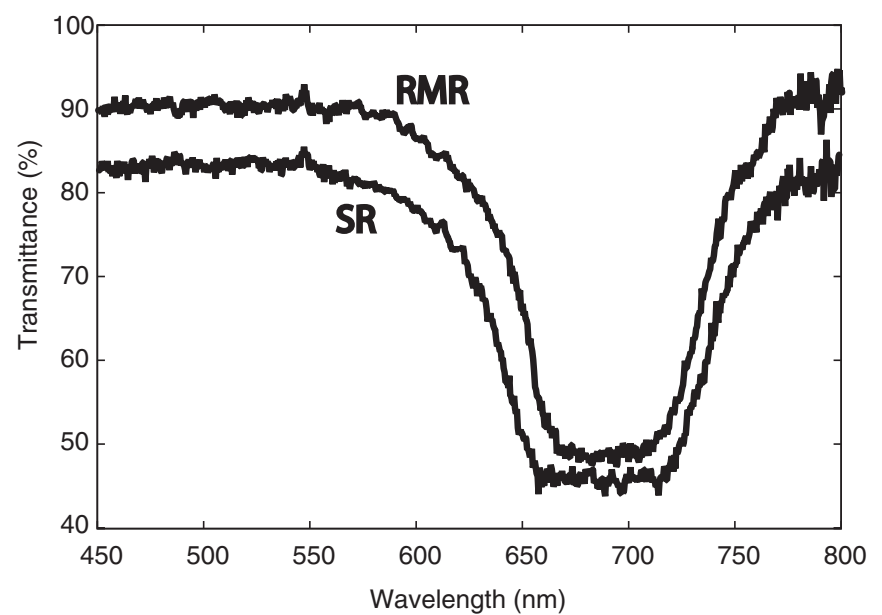

FIG. 3. Transmittance spectra of $20-\mu$ m-thick films of reactive (RMR) and nonreactive (SR) mesogens, which are the only constituents of premixture I.

and the phenomenon of broadening is confirmed in the latter one. Thin films of reactive and nonreactive mesogens prepared separately reflect light of the same wavelengths (Fig. 3). The central wavelength $\lambda_{0}$ of the band gap is directly related to the helical twisting power (HTP). The band gap width was measured between $637 \pm 5$ and $740 \pm 5 \mathrm{~nm}$ for SR, and between $646 \pm 5$ and $738 \pm 5 \mathrm{~nm}$ for RMR-which leads to $\lambda_{0}=689 \pm 5 \mathrm{~nm}$ for SR vs $\lambda_{0}=693 \pm 5 \mathrm{~nm}$ for RMR. The HTPs of both compounds are thus very similar. The broadening cannot be linked to the concentration gradient of the chiral species in a perpendicular direction to the surfaces of the cholesteric film. We mention that the small difference in transmittance spectra is due to the slightly different planar Grandjean textures; the present texture of the SR film includes more oily streaks than the RMR film, hence a light scattering effect that diminishes the transmittance was observed. In the field of polymer-stabilized cholesteric textures, it is known that the CLC material may slightly scatter the light after polymerization, which increases the number of domain boundaries and the breakup of the monodomain textures into polydomains [30]; the light scattering is extremely sensitive to defects such as disclination lines, in which monomer polymerization preferentially occurs [31].

The cross-sectional structure of the solid film-which is a crosslinked CLC polymer embedded in a CLC vitrified matrix (Sec. II)—was investigated by SEM (Fig. 4). A network of parallel stripes (so-called fingerprint texture) is displayed. The distance between two identical stripes is related to the half pitch of the helical structure. The periodicity clearly increases from the top (i.e., the side of the incoming UV beam) to the bottom (i.e., the side of the outgoing beam) of the cell. The band-gap broadening is thus connected to the creation of a pitch-gradient helical structure, which is a direct consequence of the formation of a concentration-gradient network-due to the UV absorbing properties of premixture I (Supplemental Material, Fig. S3 [27]) - and not to a concentration gradient in different chiral compounds. Asymmetrical irradiation, as commonly found in the literature, is required to generate the graded concentration in the polymer from which the

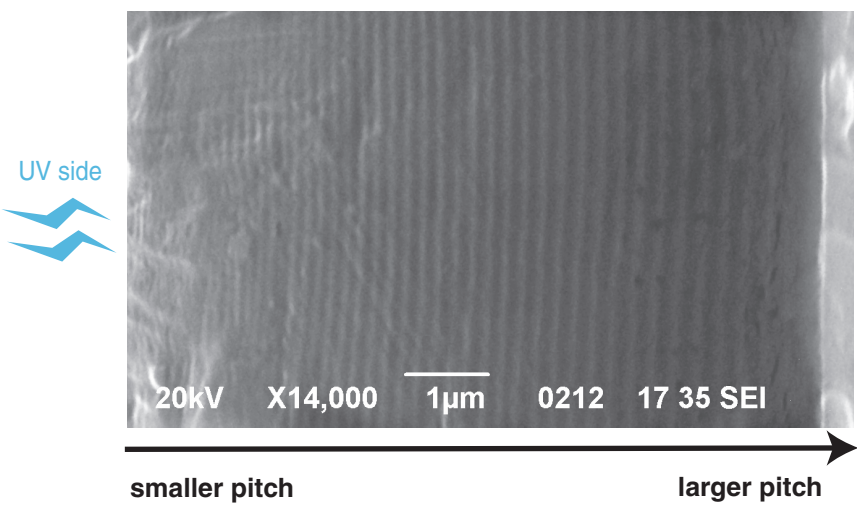

FIG. 4. (Color online) Evidence of a pitch-gradient structure in the cholesteric film of premixture I when polymerized under asymmetrical conditions. The cell was broken in a direction perpendicular to the surface of the film (Sec. II). The cross section was observed by SEM. In such a fingerprint texture, the distance between two identical stripes is directly related to the half pitch. It is thus shown that the pitch continuously increases from the left to the right side. This result is in agreement with the spring model, meaning that in the polymer-rich region (left side) the pitch is constricted whereas it is elongated in the polymer-poor region (right side).

broadening is expected. Direct reflection measurements of the cell, where a spectrophotometer measures the reflection properties of the top and bottom parts of the cell separately, yielded a clue about the distribution of the colors in the volume of the film [Fig. 2(b)]. The reflection of lower (respectively, larger) wavelengths comes from the top (respectively, bottom) of the film. The larger the local concentration in polymer is, the smaller the pitch of the gel is. This geometry is in perfect agreement with the helical structure as shown by SEM. The explanation that states the pitch is larger in regions rich in nematic compounds (a nematic structure looks like a cholesteric structure with an infinite pitch) $[4-6,8,11,13,14]$ is not valid. Most of the studies focusing on light reflection broadening as the consequence of the generation of a polymer gradient have used nematic monomers and transmission spectra. When only transmission properties are investigated, the spatial distribution of the reflection colors in the thickness of the film is not available. We believe that these experimental conditions have prevented some authors from investigating the phenomenon of mechanical stress induced by the concentration-gradient network.

\section{B. Model}

The behavior of CLC gels with a broadened band gap that is not related to a gradient in chiral species (premixture I) has been a source of inspiration for us for building a model based on a full set of structural and optical properties of premixture II. Premixture II was composed of $9 \mathrm{wt} \%$ photoreactive nematic mesogens and 91 wt $\%$ nonreactive chiral mesogens-photoinitiator mixture. The premixture fills the space between two parallel glass substrates via capillary action, forming a $6-\mu \mathrm{m}$-thick film. Our model takes into account that, after photopolymerization, the network models the helical structure of the premixture like a coil spring with an intrinsic spring constant, which depends on the polymer 


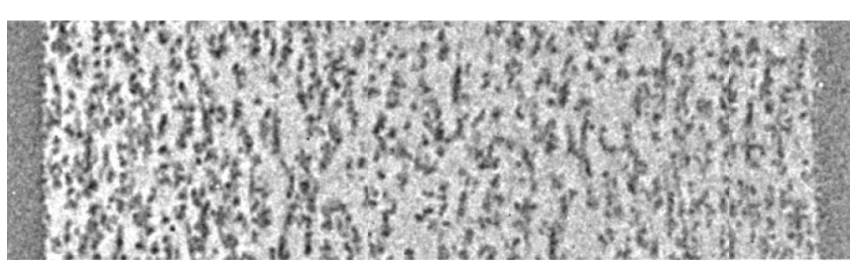

(a)

$L$
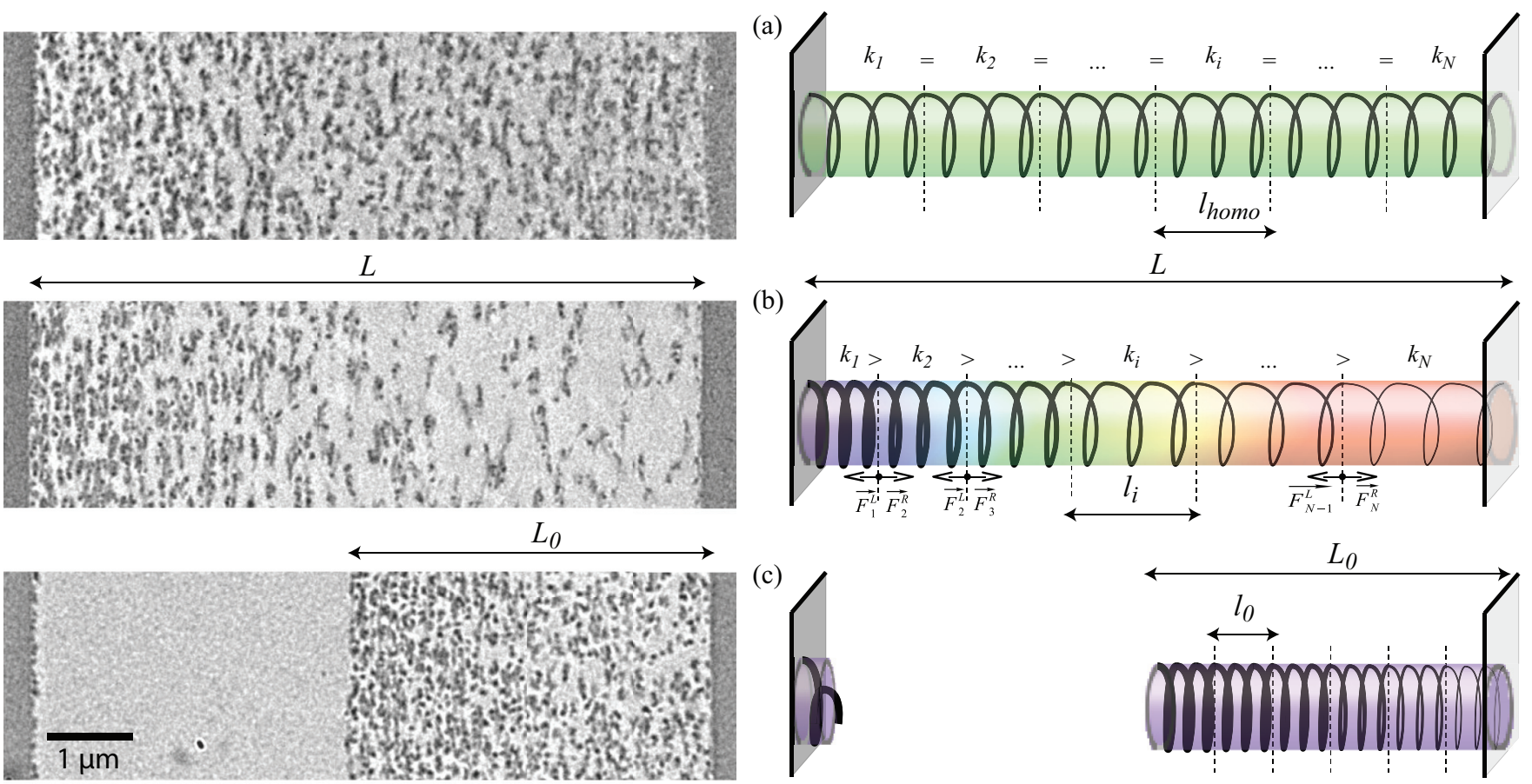

(b)

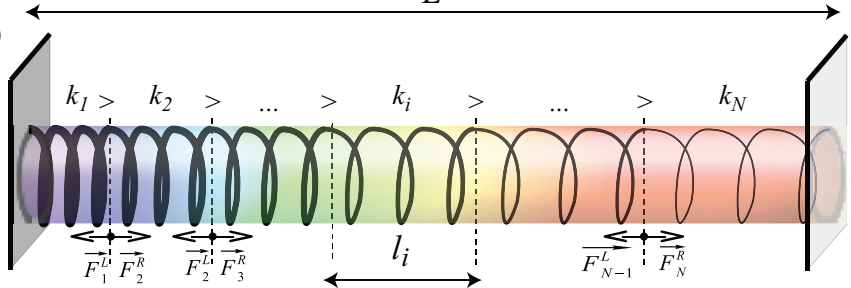

(c)

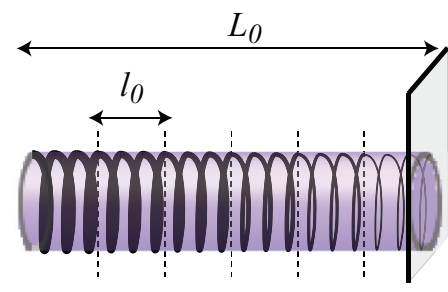

FIG. 5. (Color online) Model of coil spring with a gradient of stiffness as inspired by cross-sectional views of the polymer network elucidated by TEM. Left column: In a gel layer of premixture II, the LC was replaced with resin. The network (respectively, resin) appears dark (respectively, light grey). (a) The network concentration is homogeneously distributed in the bulk (symmetrical irradiation conditions, i.e., the cell is irradiated from both sides simultaneously). (b) A network concentration gradient is evident (asymmetrical conditions, i.e., the cell is irradiated from the left side). (c) Here the network detached from one side of the cell and relaxed into the LC volume (asymmetrical conditions). A small fraction of polymer is visible close to the left substrate. Right column: Model composed of $N$ subsprings placed end to end, which represent the helical network. The LC in which the network is embedded is represented in colors. The color code refers to the impact on the reflection color of the graded pitch. (a) All the subsprings have the same spring constant $k_{i}$ and the same length $l_{\text {homo }}$. (b) The more concentrated the network, the larger the constant of the local subspring becomes. The decreased thickness of the coil from the left part of the layer to the right one indicates the stiffness gradient of the polymer network. The spring $i$ with a length $l_{i}$ exerts a force $F_{i}^{R}$ on spring $i-l$ toward the right and a force $F_{i}^{L}$ on spring $i+l$ toward the left. In the steady state, i.e., when all the forces $F_{i}$ are the same, the springs with the smallest stiffness values (right part of the gel tube) are more elongated than the others(left part). Due to the network that models the LC structure, the pitch of the gel is proportionally distorted. (c) The subsprings relaxed and attained their unstressed configuration. All subsprings have the same length $l_{0}$.

distribution and morphology. A shrinkage effect takes place in the mixture during the polymerization process due to rearrangements of monomers (their intermolecular distances decrease) [32]. The volume contraction comes from changes in the van der Waals volume and better packing of the molecules as a result of decreased entropy within the layer [33]. Additionally, the network being formed anchors at both cell substrates. Stress intrinsic to the whole network thus arises due to the photopolymerization, the cause of which is both shrinkage and anchoring in the network. If the network detaches from a substrate, it contracts to become close to the other substrate due to the stress which is released, as we show below.

TEM investigations of the polymer distribution of premixture II for different UV irradiation cases (Fig. 5) support the model. Resin is substituted for the LC in the gel by taking care to preserve the $a b$ initio spatial distribution of the network in the cell (Sec. II). The porous network embedded in the resin is then cut in 80-nm-thick slices in a direction transverse to the film plane. The case shown in Fig. 5(a) corresponds to a symmetrical irradiation, where the premixture is polymerized from both sides of the cell by using two UV sources. The micrograph shows the network as homogeneously distributed in the resin. The gel is modeled as a spring (i.e., the helical polymer) that homogeneously models the nonreactive LC, in which it propagates from one side of the layer to the other one. The spring has the same spring stiffness, and thus the same elongation, at every point. The case shown in Fig. 5(b) corresponds to asymmetrical irradiation. The concentration gradient in the polymer is visible from the left part (i.e., the side of the incoming beam) to the right part (i.e., the side of the outgoing beam) of the layer. The stiffness of the spring decreases with the network concentration. The network looks like a coil spring with a distortion gradient. Due to the action of the graded stress, the spring is compressed in the polymer-rich region and stretched in the polymer-poor one. At the end, in the steady state, there is no more residual stress gradient in the gel layer since the different stress forces related to the different subsprings are equal. Figure 5(c) shows an especially original and interesting behavior of the spring network when it has detached from only one side of the cell and relaxed into the LC volume. A small fraction of polymer is even still visible on the left substrate side. This phenomenon of contraction is an experimental clue to the restoring force of the coil spring. 

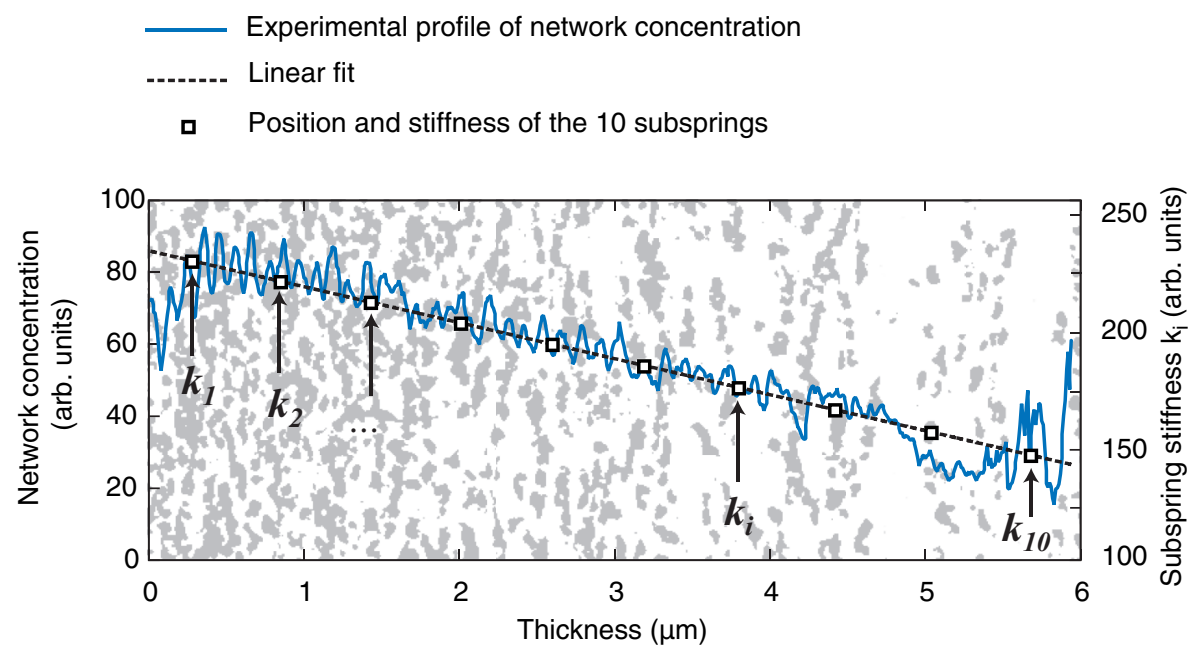

FIG. 6. (Color online) Relationship between the network concentration and the subspring stiffness in the case of asymmetrical irradiation. Solid line: experimental profile of network concentration in the direction transverse to the gel layer (premixture II) as deduced from TEM images. Dashed line: linear fit of the profile used to determine the spring constants (from $k_{1}$ to $k_{10}$ ) of ten springs uniformly distributed along the layer thickness. An example of TEM image after thresholding is given in the background.
Figure 6 shows the experimental profile of the network concentration (solid line) in the case of asymmetrical irradiation. The profile was deduced from ten TEM images after thresholding and it can be fitted by a linear function (dashed line). An example of an image after thresholding is given in the background. The coil spring model, which accounts for the graded distortion, is modeled by a series of $N$ subsprings placed one after the other.

The elongation of a given subspring depends on its force balance with the others subsprings. The local concentration in the network determines the local stiffness of each subspring. The model considers a set of subsprings with gradual variation of the stiffness from one spring to the next, which then yields the distribution in subspring elongations. In Fig. 6, we have chosen a discretization with ten subsprings. The open squares in Fig. 6 correspond to ten spring constants $k_{i}$ (right vertical scale). The location of these ten dots was uniformly distributed along the linear variation of the network concentration profile. Since the scale in network concentration (left vertical scale) is in arbitrary units (because it depends on the set of parameters chosen for the TEM image recording), the scale of subspring stiffness is also in arbitrary units. In order to determine the restoring forces of subsprings using Hooke's law, we need to know the lengths of the subsprings in their relaxed state. This information is provided in Fig. 5(c) where the network was detached from one side of the cell. The network thickness after release gives the length $L_{0}$ of the spring in its relaxed state and is found to be about $60 \%$ of the layer thickness $L$. In the case of a homogeneous network [Fig. 5(a)], the different spring constants $k_{i}$ are the same and all the subsprings have thus the same length $l_{\text {homo }}=L / 10$. In the presence of a concentrationgradient network, the stiffness $k_{i}$ of the subsprings are all different; the related lengths $l_{i}$ were calculated via Eqs. (3) and (4) (Sec. II). At the neighboring of the left side (incoming UV beam side), where the network concentration is higher, the restoring force is bigger, so the subsprings are compressed there because $l_{i}<l_{\text {homo }}$. Symmetrically, at the neighboring right side, the subsprings are extended because $l_{i}>l_{\text {homo }}$. Due to the template effect of the network on the CLC [4], the local pitch is assumed to be directly proportional to the length of the local subspring. In the region where the network concentration is higher (respectively, lower), the resulting pitch is smaller (respectively, larger) than the pitch $p_{0}$ of the premixture before photopolymerization. Reflection spectra from both sides of the cell with premixture II after polymerization confirmed that the pitch is indeed smaller close to the side of the incoming UV beam (Supplemental Material, Fig. S4 [27]). This contradicts the current explanation based on the claim that the pitch is necessarily larger in regions rich in nematic polymer, because the explanation simply assumes a correlation between the pitch variation and the variation in nematic concentration. If this explanation was right, the pitch gradient should be in the opposite direction to what is found in premixture II. In fact, the effect of the graded-stress network basically overpowers the effect of the concentration gradient in the chiral species due to the diffusion mechanism. The occurrence of this concentration gradient in chiral species, whose characteristics depend on the UV intensity and irradiation time [13], plays a role on the band-gap parameters, such as its width, height, or shape, but not on the fundamental phenomenon of broadening.

It was confirmed that, when the polymerization occurs under symmetrical conditions in premixture II, there is an absence of concentration-gradient network and band-gap broadening as well (Fig. 7). The validity of the coil spring model and its agreement with the experimental behavior when the polymerization occurs under symmetrical conditions were then confirmed.

\section{NUMERICAL SIMULATIONS}

The transmission spectrum of the pitch-gradient structure predicted by Eqs. (3) and (4) was calculated by FDTD numerical simulations (Sec. II) in order to test our model in a quantitative way. Experimental and simulated spectra are presented in Figs. 8(a) and 8(b) for premixture II. The agreement with respect to the shape, position, and width of the band gaps is very good. The only noticeable difference is the slight decrease in transmission after polymerization, which is not visible in the simulated spectrum. This is due to light scattering [30,31], which is not taken into account in the numerical simulations. The set of spring constants $k_{i}$ used to generate the simulated pitch gradient when the network concentration $C$ is equal to 9 wt \% comes from Fig. 6. Figure 8 (c) shows the remarkable nonmonotonous behavior of 
(a)

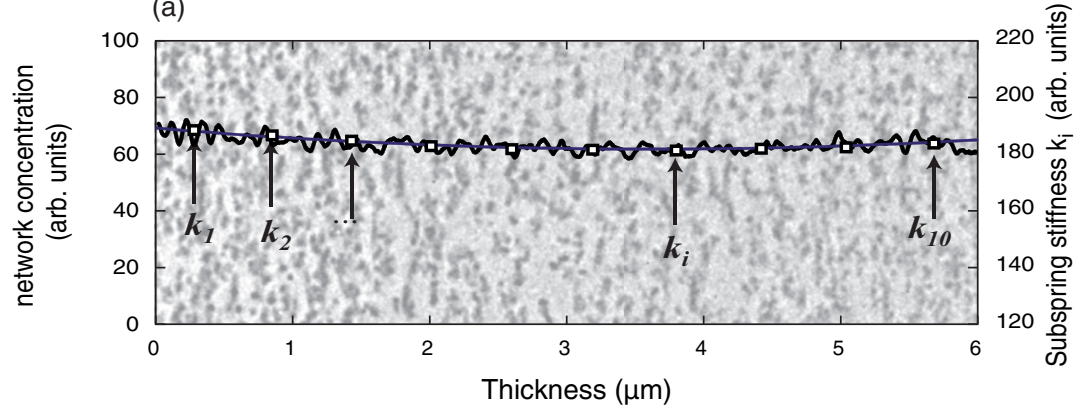

(b)

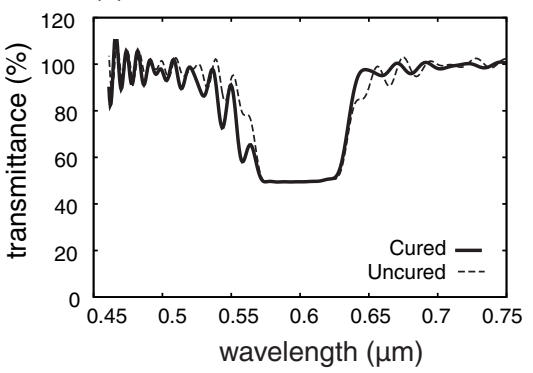

FIG. 7. (Color online) (a) TEM image of the network embedded in resin when the network is homogeneously distributed in the layer. Solid line: concentration profile as extracted from TEM images such as the image depicted in the background. Dashed line: linear fit of the profile used to determine the spring constants $k_{i}$ of ten springs uniformly distributed along the layer thickness (open squares). (b) Transmittance spectrum after curing calculated by using the coil spring model. The spectrum shows no significant broadening of the band gap, as can be seen when comparing it with the calculated spectrum before curing.

the ratio $\Delta \lambda / \Delta \lambda_{0}$ of premixture II as a function of $C$. The set of spectra used to plot Fig. 8(c) is given in Fig. S5 of the Supplemental Material [27]. This ratio is indicative of the broadening performance. For $C=0$, there is no broadening and $\Delta \lambda / \Delta \lambda_{0}=1$. As $C$ increases, the presence of the network asymmetrically distributed in the gel layer leads to the

(a)

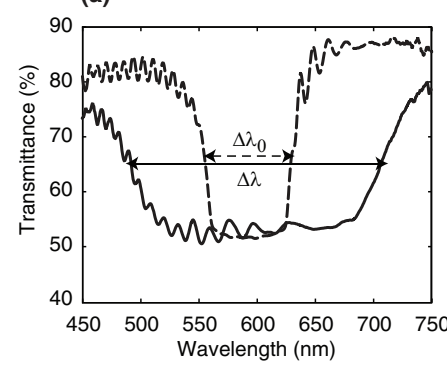

(c)

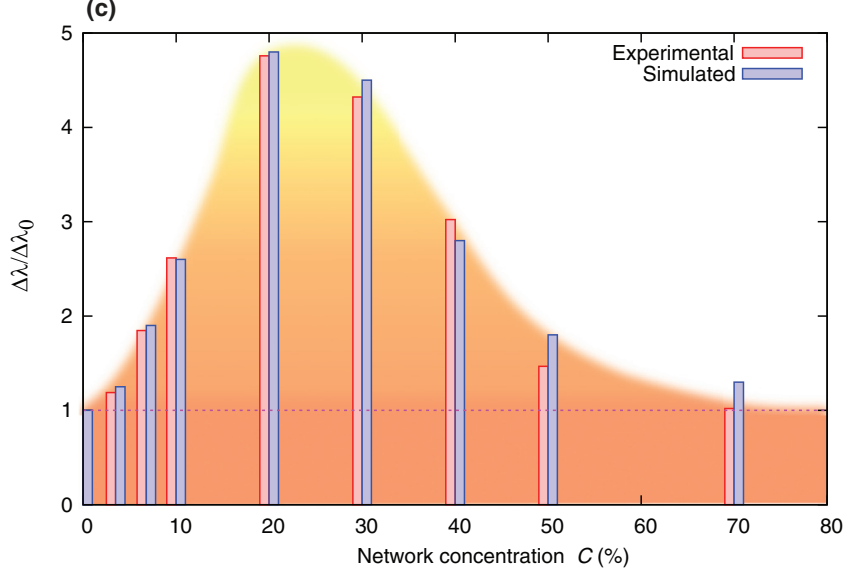

FIG. 8. (Color online) Comparison between the experimental and simulated optical behaviors, as predicted by the coil spring model. (a) Experimental and (b) simulated transmission spectra without pitch gradient (before polymerization; dashed lines) and with a pitch gradient (after polymerization; solid line). Network concentration $C=10 \%$. (c) Variation of the relative bandwidth $\Delta \lambda / \Delta \lambda_{0}$ with network concentration $C . \Delta \lambda$ (respectively, $\Delta \lambda_{0}$ ) is the full width at half maximum of the band gap after (respectively, before) polymerization. The bell-like profile is a guide for the eye. formation of a pitch gradient structure with related bandwidth broadening. The pitch gradient is enhanced when the relative percentage of reactive monomers is increased up to about $C=$ $20 \mathrm{wt} \%$. Above this critical concentration $C_{\text {crit }}$, the addition of reactive monomers induces a narrowing of the photonic band gap, and the ratio $\Delta \lambda / \Delta \lambda_{0}$ returns to 1 for $C \sim 70 \mathrm{wt} \%$. When the network concentration is increased above $C_{\text {crit }}$, the ratio $k_{i} / k_{N}$ [see Eq. (3) in Sec. II] tends toward a value of 1 and the broadening induced by the graded stress exerted by the network is not significant. The nonmonotonous behavior of the bandwidth, as shown in Fig. 8(c), is clearly reproduced by the numerical simulations. The maximal broadening is obtained when the distribution of subspring elongations is maximized. According to Eqs. (3) and (4), this situation occurs when all the $k_{i} / k_{N}$ ratios are maximized.

The curing conditions determine if whether a symmetric (Fig. 8) or nonsymmetric (Fig. 2) broadening is obtained around the central wavelength. A blueshifted nonsymmetric broadening is indeed promoted when curing occurs at very low UV light intensities and for longer times [13]. The shape of the band gap after broadening is thus related to the nature of the phase separation between the network-forming material and the nonreactive LC.

\section{CONCLUSIONS}

This paper includes two types of major advances: a methodological advance in the field of broadband cholesteric reflector-we experimentally demonstrated the broadening of the band gap in LC gels in the absence of any gradient in chiral species; a conceptual advance in the field of LC gels generally speaking-model calculations and simulations showing that the helical network acts as a coil spring with a gradient of spring constants.

In summary: (i) The broadening is the consequence of the formation of a pitch gradient. (ii) The stress gradient is at the origin of the pitch gradient. (iii) The stress is due to shrinkage during photopolymerization and the anchoring of the network at both surfaces of the cell. (iv) The stress gradient is the consequence of the existence of a stiffness gradient. (v) The stiffness gradient is the consequence of the monomer diffusion gradient. After the completion of gel formation, there is no more residual stress gradient in the gel at the steady state, since 
all stress forces related to the subsprings are equal. Whereas a gradient in the chiral species has been invoked in the literature to explain broadening, our results show that the broadening is basically caused by the graded stress provided by the coil springs. When a gradient in the chiral species does exist, its characteristics play a role on the amplitude of the broadening. The present results suggest that a revision of the physical properties of CLC gels is needed when a concentration gradient in a polymer network is present.

In view of practical applications in color display technology, the case of a CLC layer with an asymmetric structure is relevant for material implementation in a device used under asymmetrical conditions-like in intelligent (fieldswitchable) reflective cholesteric windows for energy efficiency and dynamic solar control. The physics of light propagation in a CLC gel with an asymmetric distribution in a polymer network has to be fully understood to optimize the optical and electro-optical properties of the optical layer. The present work contributes to this challenging topic by considering the basic mechanical role of the structuregradient polymer network over the whole structure of the gel, enabling an understanding of the optical behavior of the material.

\section{ACKNOWLEDGMENTS}

We thank E. Hanelt from Wacker-Chemie GmbH (Munich, Germany) for providing us with oligomers and constant support, and C. Bourgerette (from CEMES-CNRS, Toulouse) for his technical assistance with ultramicrotomy and TEM.
[1] Liquid Crystals in Complex Geometries Formed by Polymer and Porous Networks, edited by G. P. Crawford and S. Zumer (Taylor and Francis, London, 1996).

[2] I. Dierking, Adv. Mater. 12, 167 (2000).

[3] A. S. Sonin and N. A. Churochkina, Polym. Sci. Ser. A 52, 463 (2010).

[4] I. Dierking, L. L. Kosbar, A. Afzali-Ardakani, A. C. Lowe, and G. A. Held, J. Appl. Phys. 81, 3007 (1997).

[5] D. J. Broer, J. Lub, and G. N. Mol, Nature (London) 378, 467 (1995).

[6] D. Coates, M. J. Goulding, S. Greenfield, J. M. W. Hanmer, S. A. Marden, and O. L. Parri, Proc. SID 96, 67 (1996).

[7] R. A. M. Hikmet and H. Kemperman, Nature (London) 392, 476 (1998).

[8] D. J. Broer, G. N. Mol, J. A. M. M. van Haaren, and J. Lub, Adv. Mater. 11, 573 (1999)

[9] S. Relaix, C. Bourgerette, and M. Mitov, Appl. Phys. Lett. 89, 251907 (2006).

[10] S. Relaix, C. Bourgerette, and M. Mitov, Liq. Cryst. 34, 1009 (2007).

[11] B. Fan, S. Vartak, J. N. Eakin, and S. M. Faris, Appl. Phys. Lett. 92, 061101 (2008).

[12] A. Castellanos-Moreno, P. Castro-Garay, S. Gutierrez-Lopez, R. A. Rosas-Burgos, A. Corella-Madueno, and J. A. Reyes, J. Appl. Phys. 106, 023102 (2009).

[13] L. V. Natarajan, J. R. Voss, V. P. Tondiglia, D.-K. Yang, T. J. White, and T. J. Bunning, Proc. SPIE 7775, $77750 Z$ (2010).

[14] K.-S. Bae, U. Cha, Y.-J. Lee, Y.-K. Moon, H.-C. Choi, J.-H. Kim, and C.-J. Yu, Opt. Express 19, 8291 (2011).

[15] T. J. White, S. A. Cazzell, A. S. Freer, D.-K. Yang, L. Sukhomlinova, L. Su, T. Kosa, B. Taheri, and T. J. Bunning, Adv. Mater. 23, 1389 (2011).

[16] C. A. Guymon, E. N. Hoggan, N. A. Clark, T. P. Rieker, D. M. Walba, and C. N. Bowman, Science 275, 57 (1997).
[17] H. Kikuchi, M. Yokota, Y. Hisakado, H. Yang, and T. Kajiyama, Nat. Mater. 1, 64 (2002)

[18] F. Castles, F. V. Day, S. M. Morris, D.-H. Ko, D. J. Gardiner, M. M. Qasim, S. Nosheen, P. J. W. Hands, S. S. Choi, R. H. Friend, and H. J. Coles, Nat. Mater. 11, 599 (2012).

[19] M. Mitov and N. Dessaud, Nat. Mater. 5, 361 (2006).

[20] B. Park et al., Adv. Mater. 21, 771 (2009).

[21] H. Kelker and R. Hatz, in Handbook of Liquid Crystals (Verlag Chemie, Weinheim, 1980), pp. 293-337.

[22] Liquid Crystals-Frontiers in Biomedical Applications, edited by S. J. Woltman, G. D. Jay, and G. P. Crawford (World Scientific, Singapore, 2007).

[23] M. Mitov, Adv. Mater. 24, 6260 (2012).

[24] F. Ardavan, D. R. Oskooi, M. Ibanescu, P. Bermel, J. D. Joannopoulos, and S. G. Johnson, Comput. Phys. Commun. 181, 687 (2010).

[25] F.-H. Kreuzer, N. Häberle, H. Leigeber, R. Maurer, J. Stohrer, and J. Weiss, in Organosilicon Chemistry III, edited by N. Auner and J. Weiss (Wiley-VCH, New York, 1997), pp. 566-586.

[26] G. Agez and M. Mitov, J. Phys. Chem. B 115, 6421 (2011).

[27] See Supplemental Material at http://link.aps.org/supplemental/ 10.1103/PhysRevE.89.022513 for Figs. S1-S5.

[28] D. J. Broer and I. Heynderickx, Macromolecules 23, 2474 (1990).

[29] M. Mitov and N. Dessaud, Liq. Cryst. 34, 183 (2007).

[30] D. J. Dyer, U. P. Schröder, K.-P. Chan, and R. J. Twieg, Chem. Mater. 9, 1665 (1997).

[31] R. Borsali, U. P. Schroeder, D. Y. Yoon, and R. Pecora, Phys. Rev. E 58, R2717 (1998).

[32] R. A. M. Hikmet and J. Lub, Prog. Polym. Sci. 21, 1165 (1996).

[33] R. A. M. Hikmet, B. H. Zwerver, and D. J. Broer, Polymer 33, 89 (1992). 